\title{
X-ray imaging of the ionisation cones in NGC 5252
}

\author{
M. Dadina ${ }^{1,2}$, M. Guainazzi ${ }^{3}$, M. Cappi ${ }^{1}$, S. Bianchi ${ }^{4}$, C. Vignali ${ }^{2}$, G. Malaguti ${ }^{1}$, and A. Comastri ${ }^{5}$ \\ 1 INAF/IASF Bologna, via Gobetti 101, 40129 Bologna, Italy \\ e-mail: dadina@iasfbo.inaf.it \\ 2 Università degli Studi di Bologna, Dip. di Astronomia, via Ranzani 1, 40127 Bologna, Italy \\ 3 European Space Astronomy Center of ESA, Apartado 50727, 28080 Madrid, Spain \\ 4 Dipartimento di Fisica, Università degli Studi Roma Tre, via della Vasca Navale 84, 00046 Roma, Italy \\ 5 INAF/Osservatorio Astronomico di Bologna, via Ranzani 1, 40127 Bologna, Italy
}

Received 24 November 2009 / Accepted 6 March 2010

\section{ABSTRACT}

\begin{abstract}
Context. The physical conditions of the gas forming the narrow-line regions (NLR) in active galactic nuclei (AGN) have been extensively studied in the optical band. Recently detailed X-ray studies have shown how the emission in the $0.1-2 \mathrm{keV}$ band detected in Seyfert 2 galaxies is associated to gas lying close to or associated with the NLR.

Aims. We take advantage of the spectacular extension $\left(\sim 15^{\prime \prime}\right)$ of the NLR in the type II Seyfert galaxy NGC 5252 and of the complementary characteristics of XMM-Newton and Chandra to investigate the physical conditions of the gas in this galaxy.

Methods. The X-ray data from XMM-Newton are used to define the spectral properties of the ionising nuclear source. The Chandra data are used to trace the spatial characteristics of the soft X-ray emission. This information is then compared to the optical HST characteristics of the NLR in NGC 5252.

Results. The X-ray spectrum of the nucleus of NGC 5252 is intrinsically flat $(\Gamma \sim 1.4-1.5)$ and absorbed by neutral gas with a column density $N_{\mathrm{H}} \sim 10^{22} \mathrm{~cm}^{-2}$. Below $\sim 1 \mathrm{keV}$ a soft excess is detected. The high-resolution spectrum obtained with the XMM-Newton RGS shows emission lines in the $0.2-1.5 \mathrm{keV}$ range which strongly indicate that the soft $\mathrm{X}$-ray component is essentially due to ionised gas. Moreover, the soft X-ray emission is spatially resolved around the nucleus and well overlaps the images obtained in narrow optical bands centred around the [OIII] emission line at $5007 \AA$. The [OIII]/soft-X flux ratios along the ionisation cones are basically constant. This indicates that the electron density does not significantly deviate from the $r^{-2}$ law (constant ionisation parameter) moving outward from the nucleus.

Conclusions. This result combined with previous optical studies suggests two plausible but different scenarios in the reconstruction of the last $\sim 30000$ years of history of the central AGN. The most promising one is that the source is indeed a "quasar relic" with a steady and inefficient energy release from the accretion of matter onto the central super-massive black-hole. This scenario is suggested also by the flat nuclear X-ray spectrum that evokes an advection dominated accretion flow (ADAF) like emission mechanism.
\end{abstract}

Key words. galaxies: active - galaxies: individual: NGC 5252 - galaxies: Seyfert - X-ray: galaxies

\section{Introduction}

The obscuration of the nuclear emission in type II AGN allows the study of soft X-ray spectral components, which are normally outshone by the direct component in type I unobscured objects. It has been well known since the early days of X-ray spectroscopy that excess emission above the extrapolation of the absorbed nuclear radiation is present in almost all bright Seyfert $2 \mathrm{~s}$ (Turner et al. 1997). This excess appears smooth when measured with instruments with moderate energy resolutions like CCD. However, high-resolution (grating) measurements with Chandra and XMM-Newton revealed that this excess is generally due to a blending of strong recombination lines from $\mathrm{He}$ - and $\mathrm{H}$-like transitions of elements from carbon to nitrogen (Sako et al. 2000; Sambruna et al. 2001; Kinkhabwala et al. 2002; Armentrout et al. 2007). X-ray spectral diagnostics (Kinkhabwala et al. 2002; Guainazzi \& Bianchi 2006) and a close morphological coincidence between the soft X-rays and the [OIII] in extended narrowline regions (ENLR; Bianchi et al. 2006, 2010) strongly indicate that the gas is photoionised by the AGN, with an important role played by resonant scattering.

In this context, NGC 5252 represents an extraordinary laboratory to study the feedback between the AGN output and circumnuclear gas on kpc scale because of its spectacular ionisation cones (Tadhunter \& Tsvetanov 1989).

The source NGC 5252 is classified as a Seyfert 1.9 (Osterbrock \& Martel 1993) S0 (de Vaucoulers et al. 1991) nearby $\left(z=0.023\right.$, ) galaxy $\left(N_{\mathrm{H}, \mathrm{Gal}}=2.14 \times 10^{+20} \mathrm{~cm}^{-2}\right.$, Dickey $\&$ Lockman 1990). Small radio jets $\left(r \sim 4^{\prime \prime}\right)$ have been detected and found to be aligned with the ionisation cones (Wilson \& Tsvetanov 1994). Nonetheless, the host galaxy luminosity $\left(M_{R} \sim-22\right.$, Capetti et al. 2005), mass $\left(M_{\text {bulge }} \sim 2.4 \times 10^{11} M_{\odot}\right.$, Marconi \& Hunt 2003) and the mass of the central super-massive black-hole $\left(M_{\mathrm{BH}} \sim 10^{9} M_{\odot}\right.$, Capetti et al. 2005 are more typical of quasar than Seyfert galaxies. These pieces of evidence led Capetti et al. 2005) to speculate that NGC 5252 is most probably to be considered a QSO relic. This agrees with "downsizing" scenarios about the evolution of super-massive black-hole (SMBH) in cosmic times. According to these scenarios, most massive SMBHs formed and evolved earlier than lower mass ones.

Ionisation cones are one of the strongest arguments in favour of the Seyfert unification scenarios (Antonucci 1993). For this reason, NGC 5252 is also an important laboratory to test AGN geometrical models. From a diferent point of view, AGN activity has been recognized, for a while as a key component of the 
SMBH host galaxy co-evolution and AGN feedback is likely to self-regulate or be responsible for the observed properties (Menci et al. 2004). The very existence of ionisation cones is a witness that feedback/winds are or were active and thus these sources are ideal laboratories for feedback.

$\mathrm{X}$-ray measurements allow us to directly link the properties of the gas emitting optical lines with the intrinsic AGN power, which in type II AGN can be truly measured only at energies higher than the soft photoelectric cut-off due to the AGN obscuring matter. Furthermore, the morphological coincidence between X-rays and optical emission in ENLR (Bianchi et al. 2006) points to a fundamental physical link between the two wavebands. They need to be studied simultaneously to derive the correct energy budget in the ionisation cones. Prompted by these motivations, we have performed deep X-ray observations of NGC 5252 at the highest spatial and spectral resolution currently available with Chandra and XMM-Newton. The results of these observations are the subject of this paper.

\section{The nuclear spectrum}

NGC 5252 was observed by XMM-Newton on 2003, July 18th, with the EPIC CCDs (MOS and pn in full window, see Table 1) as the prime instrument for a total duration of $\sim 67 \mathrm{ks}$. The Observation Data Files (ODFs) were reduced and analysed with the latest Science Analysis System (SAS) software package (Gabriel et al. 2003) with the associated latest calibration files. We used the epproc and emproc tasks to generate event files and remove dead and hot pixels. Several time intervals with a high background rate were identified in the single events light curve at energies $>10 \mathrm{keV}$ and were removed, yielding a net exposure of $\sim 50 \mathrm{ks}$ for the MOS and $\sim 38 \mathrm{ks}$ for the pn. Pile-up is negligible in this source according to the epatplot SAS task outcome. Patterns $\leq 12$ and $\leq 4$ were considered for MOS and pn, respectively. The source counts were extracted from a circular region with radius $50^{\prime \prime}$, thus encompassing a large fraction of the optically-defined galaxy. The background was estimated with both blank-sky files and locally from a offset source-free region. Light curves in the soft $(0.5-2 \mathrm{keV})$ and hard $(2-10 \mathrm{keV})$ energy bands were first investigated. We found no significant flux nor spectral variations, thus considered the time averaged spectrum.

The best-fit spectrum is shown in Fig. 1. If the nuclear emission is modelled with a simple absorbed power-law we obtain an extremely flat photon index $\left[\Gamma=1.05 \pm 0.10\right.$ and $N_{\mathrm{H}}=$ $(2.2 \pm 0.1) \times 10^{22} \mathrm{~cm}^{-2}$, the reported errors are, here and hereafter, at $90 \%$ confidence level], plus a soft component emerging at energies below $E \sim 1 \mathrm{keV}$. It can be parameterised with a scattered power-law with a steep photon index $\Gamma=3.0 \pm 0.2$. There is also evidence for one (or few) soft emission lines in addition to the soft continuum, a Fe $\mathrm{K}_{\alpha}$ line with $E=6.44 \pm 0.05 \mathrm{keV}$ and $E W=50 \pm 25 \mathrm{eV}$, and an absorption edge at $E=7.0 \pm 0.1 \mathrm{keV}$ and optical depth $\tau=0.31 \pm 0.05$. A significantly better fit is obtained for a power-law plus a thermal component, namely mekal in Xspec (Mewe et al. 1985), which is used to model the soft X-ray band of NGC $5252\left(\chi^{2} /\right.$ d.o.f. $=1436 / 1410$ in the first case while $\chi^{2} /$ d.o.f. $=1329 / 1410$ with mekal $)$. In this case, the temperature of the plasma is $k T \sim 0.17 \mathrm{keV}$. The investigation on the true nature of this soft X-ray component will be the subject of the next sections. Here it is important to note that whatever the fitting of the data below $\sim 1 \mathrm{keV}$, the best-fit model for the nuclear emission of NGC 5252 above $\sim 1 \mathrm{keV}$ is typical in shape, but flatter $(\Gamma \sim 1.4-1.5)$ than normally found from Seyfert 2 galaxies $(\Gamma=1.5-2.5$, Turner \& Pounds 1989; Turner et al. 1997;
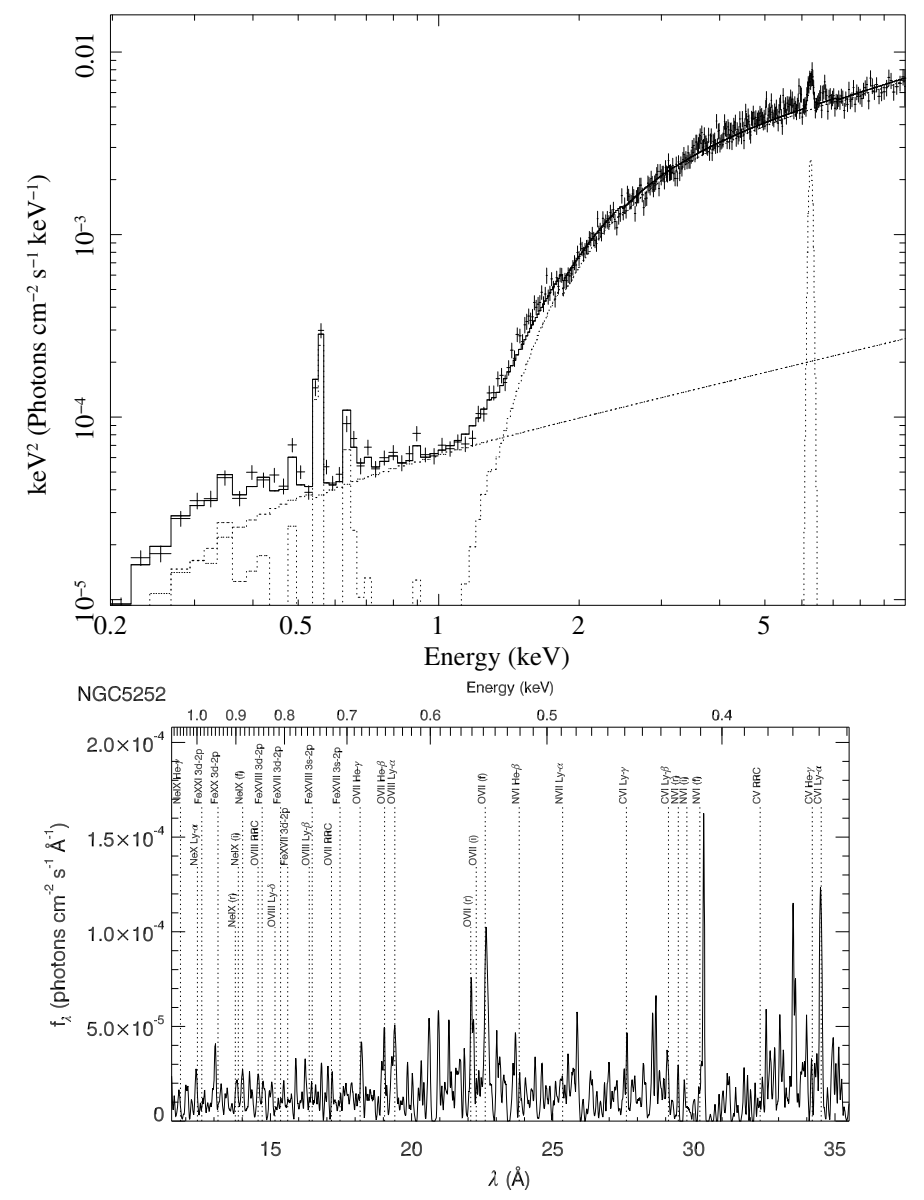

Fig. 1. Upper panel: XMM-Newton EPIC spectrum extracted from a $50^{\prime \prime}$ region around the NGC 5252 nucleus. For clarity, only pn data are presented. Lower panel: XMM-Newton RGS spectrum of NGC 5252.

Risaliti 2002; Cappi et al. 2006; Dadina 2008). It is however consistent with the previous ASCA measurement $(\Gamma \sim 1-$ 1.5, Cappi et al. 1996), confirming the need in this source for a more complex absorption (either multiple, ionised or both) to recover a steeper canonical photon index. With the above model we measure for the soft $(0.5-2 \mathrm{keV})$ component a flux of $3.5 \times 10^{-13} \mathrm{erg} \mathrm{cm}^{-2} \mathrm{~s}^{-1}$ corresponding to a luminosity of $4.1 \times 10^{41} \mathrm{erg} / \mathrm{s}$ and for the hard $(2-10 \mathrm{keV})$ component a flux of $8.9 \times 10^{-12} \mathrm{erg} \mathrm{cm}^{-2} \mathrm{~s}^{-1}$ corresponding to a (unabsorbed) luminosity of $1.2 \times 10^{43} \mathrm{erg} / \mathrm{s}$. This is consistent, within a few tens of percent, with previous ASCA and BeppoSAX values (Cappi et al. 1996; Dadina 2007). It is worth noting here that from IR diagnostics we expect that star-forming activity should contribute less than $\sim 1 \%$ to the total soft X-ray emission (Cappi et al. 1996).

\section{High-resolution spectroscopy of the AGN environment}

The Reflection Grating Spectrometer (RGS; der Herder et al. 2001) on board XMM-Newton observed NGC 5252 simultaneously with the EPIC cameras (Table 1, Fig. 1). It produces highresolution (first order resolution $600-1700 \mathrm{~km} \mathrm{~s}^{-1}$ ) spectra in the $6-35 \AA(0.35-2 \mathrm{keV})$ range. Its $2.5^{\prime}$ diameter slit fully encompasses the ionisation cones and the host galaxy. The RGS spectrum represents therefore only the average conditions of the soft X-ray emitting gas across the nucleus and the cone. 
M. Dadina et al.: X-ray imaging of the ionisation cones in NGC 5252

NGC5252 - Chandra ACIS-S

$0.2-1 \mathrm{keV}$

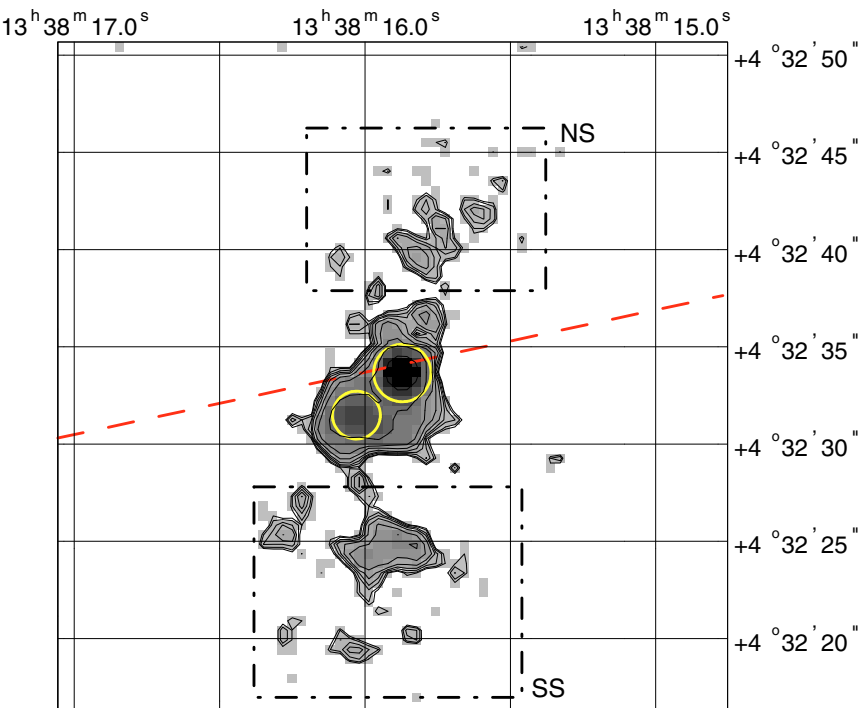

NGC5252 - Chandra ACIS-S

$1-10 \mathrm{keV}$

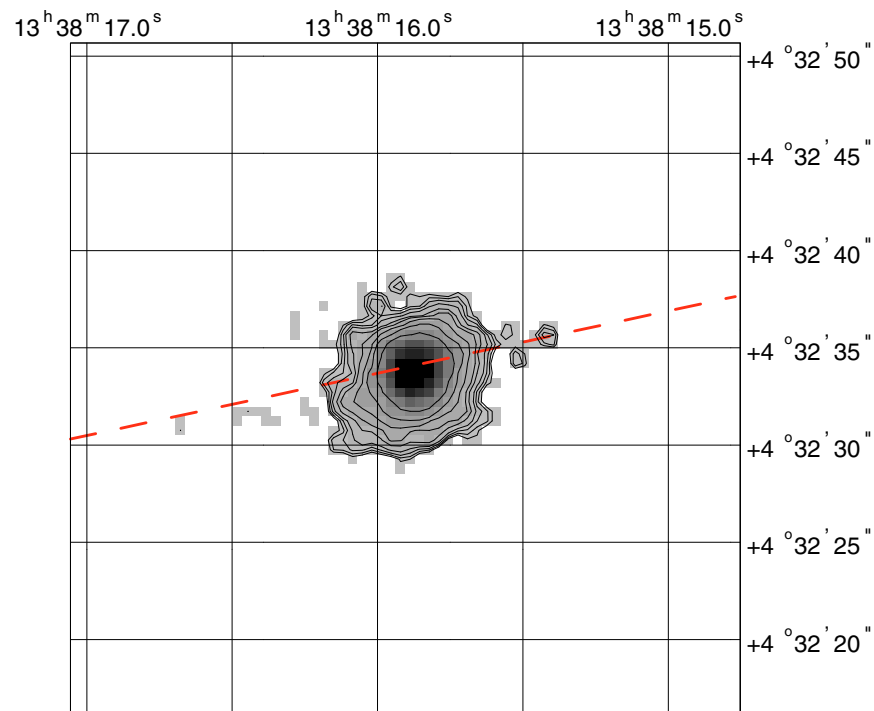

Fig. 2. Chandra ACIS-S images of NGC 5252 in the $0.1-1 \mathrm{keV}$ (soft; left panel), and 1-10 keV (hard; right panel). The images were smoothed with a $\sigma=1.25$ pixel (yielding an angular resolution of $\simeq 1^{\prime \prime}$ in the images) wavelet for illustration purposes. The thin solid lines represent nine linearly spaced contours in the range of 2 to 20 counts per pixel. The thick dot-dashed line indicates the position of the out-of-time events readout streak, removed before the generation of the image. The dashed lines represents the regions whence the spectra of the south and north diffuse spots were extracted. The solid circles represent the regions whence the spectra of the nucleus (the brightest and central spot) and of the south-east nuclear source were extracted.

Table 1. Main characteristics of the X-ray observations presented here.

\begin{tabular}{lrrlrc}
\hline \hline Instr. $^{\star}$ & $\begin{array}{r}\text { Exp. }^{\dagger} \\
\text { ks }\end{array}$ & $\begin{array}{c}\mathrm{CR}^{\ddagger} \\
\text { c/s }\end{array}$ & Instr. $^{\star}$ & $\begin{array}{r}\text { Exp. } \\
\text { ks }\end{array}$ & $\begin{array}{c}\mathrm{CR}^{\ddagger} \\
\mathrm{c} / \mathrm{s}\end{array}$ \\
\hline Epn & 38 & $1.10^{a}$ & RGS1 & 63 & $0.08^{b}$ \\
EMOS1 & 49 & $0.37^{a}$ & RGS2 & 63 & $0.08^{b}$ \\
EMOS2 & 50 & $0.37^{a}$ & ACIS-S & 60 & $0.32^{a}$ \\
\hline
\end{tabular}

Notes. ${ }^{(\star)}$ Detector name; $\left.{ }^{(}\right)$net exposure; ${ }^{(*)}$ measured count-rate; (a) count-rate in the $0.2-10 \mathrm{keV}$ band; ${ }^{(b)}$ count-rate in the $0.4-1.2 \mathrm{keV}$.

The RGS data were reduced starting from the Observation Data Files with SASv6.5 (Gabriel et al. 2003) and using the latest calibration files. The SAS meta-task rgsproc was used to generate source and background spectra, assuming as a reference coordinate coincident with the optical nucleus of NGC 5252. Background spectra were generated with both blank field maps - accumulated across the whole mission - and a "local" background accumulated during the observation. The former overestimates the intrinsic background level during the observation and is based on a model of the estimated background on the basis of the count rates detected in the most external of the camera CCDs. We therefore employed the "local" background hereafter. A correction factor to the count background spectrum was applied to take the size of the extraction region into account, which corresponds to the area of the RGS active CCDs outside the $98 \%$ percentage point of the line spread function in the cross-dispersion direction.

We simultaneously fitted the spectra of the two cameras following the procedure outlined in Guainazzi \& Bianchi $(2006)^{1}$ who performed local spectral fits around each of the

1 This paper discusses a sample of 69 RGS spectra of type 1.5, 1.8, 1.9 and 2 Seyfert galaxies. The observation of NGC 5252 discussed in this paper belongs to this sample as well.

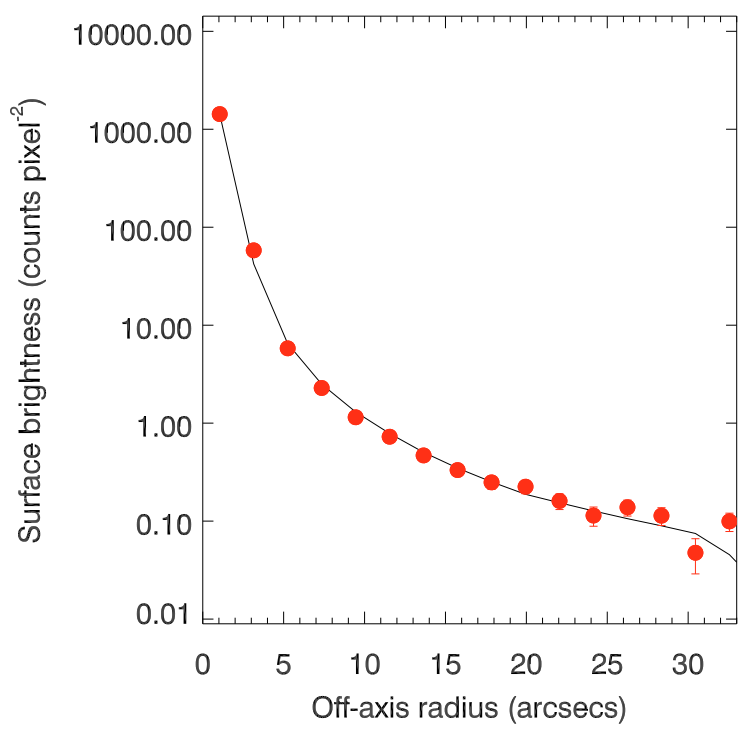

Fig. 3. Radial profile (filled circles) of the ACIS-S hard-band image. The solid line represents the PSF for a source with the same hard X-ray spectral energy distribution as the NGC 5252 "nucleus normalized" to its on-axis peak flux. When not visible, the error bars are within the filled circles.

$\simeq 40$ emission lines detected in the archetypal obscured Seyfert NGC 1068 (Kinkhabwala et al. 2002). In these fits, both the background level and the continuum were assumed as independent power-law components with the photon index $\Gamma$ set equal to 1 . It is worth noting that the adopted value of the power law index, here equal to the photon index of the continuum of the primary emission, does not affect the results signifcantly, given 
Table 2. List of emission lines detected in the RGS spectrum of NGC 5252.

\begin{tabular}{lcccc}
\hline \hline Identification & $\begin{array}{c}E_{c}{ }^{a} \\
(\mathrm{eV})\end{array}$ & $\begin{array}{c}L^{b} \\
\left(10^{40} \mathrm{erg} \mathrm{s}^{-1}\right)\end{array}$ & $\Delta v^{c}$ & $v_{\text {sys }}{ }^{d}$ \\
\hline CVI Ly- $\alpha$ & $367 \pm 5$ & $3 \pm_{2}^{11}$ & $800 \pm 400$ & 70 \\
OVII He- $\alpha(\mathrm{f})$ & $560.4 \pm 0.2$ & $2.0 \pm_{0.9}^{0.8}$ & $-370 \pm 110$ & 110 \\
OVII He- $\alpha(\mathrm{i})$ & $E_{\mathrm{c}}(f)+7.7$ & $<0.9$ & & \\
OVII He- $\alpha(\mathrm{r})$ & $E_{\mathrm{c}}(f)+13.0$ & $<1.4$ & & \\
OVIII Ly- $\alpha$ & $654.0 \pm_{1.2}^{0.7}$ & $0.6 \pm 0.4$ & $300 \pm 000$ & 130 \\
\hline
\end{tabular}

Notes. ${ }^{(a)}$ Measured line energy; ${ }^{(b)}$ intrinsic line luminosity; ${ }^{(c)}$ difference between the measured line energy and the laboratory energy expressed in $\mathrm{km} \mathrm{s}^{-1}$. Only statistical errors on this measurements are quoted; ${ }^{(d)}$ systematic error on $\Delta v$ expressed in $\mathrm{km} \mathrm{s}^{-1}$ due to residual uncertainties in the RGS aspect solution $(\simeq 8 \mathrm{~m} \AA)$.

the very limited band of these fits. Different choices for the continuum spectral index yield indistinguishable results. Each emission line was modelled with an unresolved Gaussian profile fixed to be at the expected energies (leaving the intrinsic width of the profile free yields a negligible improvement in the quality of the fit). We detect three lines (see lower panel of Fig. 1) at a confidence level larger than $90 \%\left[\Delta \chi^{2}=10.5,24.0,10.8\right.$ for $\mathrm{CV}$, OVII and OVIII lines, respectively, for one interesting parameter (Table 2)].

None of them is a radiative recombination rontinuum (RRC). A (admittedly loose) constraint on the width of the Gaussian profile can be obtained on the OVII He- $\alpha$ triplet only: $\sigma<$ $4400 \mathrm{~km} \mathrm{~s}^{-1}(8.2 \mathrm{eV})$. Diagnostic parameters involving the intensity of the OVII He- $\alpha$ triplets can be used in principle to pinpoint the physical process responsible for the bulk of the X-ray emission in high-resolution spectra. The detection of the forbidden $(f)$ component only allows us to set lower limits on the standard triplet diagnostics (Gabriel \& Jordan 1969; Porquet \& Dubau 2000): $R>1.1, G>0.7$ (where $R$ is the ratio between forbidden and intercobination lines and depends on the electron density, while $G$ is the ratio between intercombination plus forbidden lines and the resonance line, Gabriel \& Jordan 1969; Porquet \& Dubau 2000).

These limits, although fully consistent with photoionised plasmas, do not rule out collisional ionisation. Guainazzi \& Bianchi (2007) proposed a criterion to distinguish on a statistical basis between AGN- and starburst-powered sources based on the location of the source in an empirical observable plane: integrated luminosity of the $\mathrm{He}$ - and $\mathrm{H}$-like oxygen lines, $L_{\mathrm{O}}$, against the intensity ratio $\eta$ between the $f$ and the OVIII Ly$\alpha$. In NGC $5252 \eta=2.3 \pm 0.4$, and $L_{0} \sim 3 \times 10^{40} \mathrm{erg} \mathrm{s}^{-1}$. These values put NGC 5252 in the plane locus preferentially occupied by photoionised (AGN) sources (Guainazzi et al. 2009). We also estimated the flux density associated to the continuum, using a line-free energy range between 586 and $606 \mathrm{eV}$ : $\left.v L_{v}\right|_{0.6 \mathrm{keV}}=\left(7.2 \pm_{2.9}^{1.7}\right) \times 10^{40} \mathrm{erg} \mathrm{s}^{-1}$.

\section{X-ray imaging of the ionisation cones}

Chandra observed NGC 5252 on August 11, 2003 with the ACIS-S detector in standard VFAINT configuration. Data reduction was performed with CIAO version 3.3 and associated CALDB version 3.2. "Level 1" events were corrected for bad pixels, gain spatial dependency, and charge transfer inefficiency via acis_process_events.
Although the correction for read-out streaks was applied as well, some out-of-time events remained in the final cleaned event list, and were removed by applying a 2 pixels $\left(\simeq 1^{\prime \prime}\right)$-wide tilted rectangular box around the streak.

The ACIS-S images in the $\sim 2^{\prime}$ around the optical core of NGC 5252 are shown in Fig. 2 in the $0.2-1 \mathrm{keV}$ and in the 1-10 keV energy bands. The soft band clearly shows extended emission in the north-south direction on both sides of the nucleus. On the contrary, the hard band image is point-like. We extracted a radial profile of the latter and compared it with the expected instrumental point spread function (PSF) of a source with the same spectral energy distribution as the NGC 5252 nucleus. The two profiles are perfectly consistent up to $30^{\prime \prime}$ off-axis (see Fig. 3).

In order to characterize the spectral behaviour of the diffuse emission, we extracted spectra from the four regions, identified in the soft image (Fig. 2): the nucleus (N), a S-E source about $3.2^{\prime \prime}$ from the nucleus (SENS), and the South (SS) and North (NS) diffuse Spots. Background spectra were generated from a large circle $57^{\prime \prime}$ wide around the galaxy core, once a $21^{\prime \prime}$ inner circle, as well as $5^{\prime \prime}$ circles around each serendipitous point sources were removed. Alternative choices of the background regions do not substantially change the results presented in this section. Source spectra were rebinned to over-sample the intrinsic instrumental energy resolution by a factor $\geq 3$, and to have at least 25 background-subtracted counts in each spectral bin. The latter criterion ensures the applicability of the $\chi^{2}$ statistics.

For all spectra we employed a baseline model that include a thermal emission component from collisionally excited plasma (mekal in XsPEC; Mewe et al 1985). This choice was done for simplicity and the only information obtained using mekal is the flux of the thermal component. This is particularly true for the SS and NS regions where the complexity of the mekal model is well above the quality of the data. Moreover, a photoelectricallyabsorbed power-law was always included in the data. The physical meaning of the latter varies depending on the region in which the spectrum was extracted. For the nuclear region, the non-thermal component represents the contribution of the active nucleus; for the other regions, the integrated contribution of hard galactic sources like for example X-ray binaries, cataclysmic variables or supernova remnants. We therefore refrain from attributing a physical meaning to the power-law spectral indeces in the latter case. The spectra and corresponding bestfits are shown in Fig. 4. A summary of the spectral results is presented in Table 3. The Chandra data confirm that the nuclear spectrum is remarkably flat and is seen through a substantial column density $\left(N_{\mathrm{H}} \simeq 2.26 \times 10^{22} \mathrm{~cm}^{-2}\right)$.

The background subtraction for the spectrum of SENS could be contaminated by the spilling of the nuclear emission. The encircled energy fraction at a distance equal to that between sources $\mathrm{N}$ and $\mathrm{SENS}$ is $\simeq 97.5 \%$. However, subtracting a properly rescaled nuclear spectrum to the SENS spectrum yields negative counts above $2 \mathrm{keV}$. In order to have an independent estimate of the spectral behaviour of SENS, we extracted images $10^{\prime \prime}$ around the nuclear region in narrow energy bands (Fig. 5): 200-400 eV, 500-600 eV, 600-700 eV and 700-1000 eV. In a line-dominated plasma, the above energy ranges correspond to bands dominated by $\mathrm{CVI}$ and $\mathrm{CV} \mathrm{K}_{\alpha}$, OVII He- $\alpha$, OVIII Ly- $\alpha$, and Fe-L transitions, respectively. Each image was normalized to the peak of the nuclear emission in that energy band. The soft X-ray SENS spectrum is comparatively dominated by oxygen transitions, with little contribution in either the carbon or the iron band. 

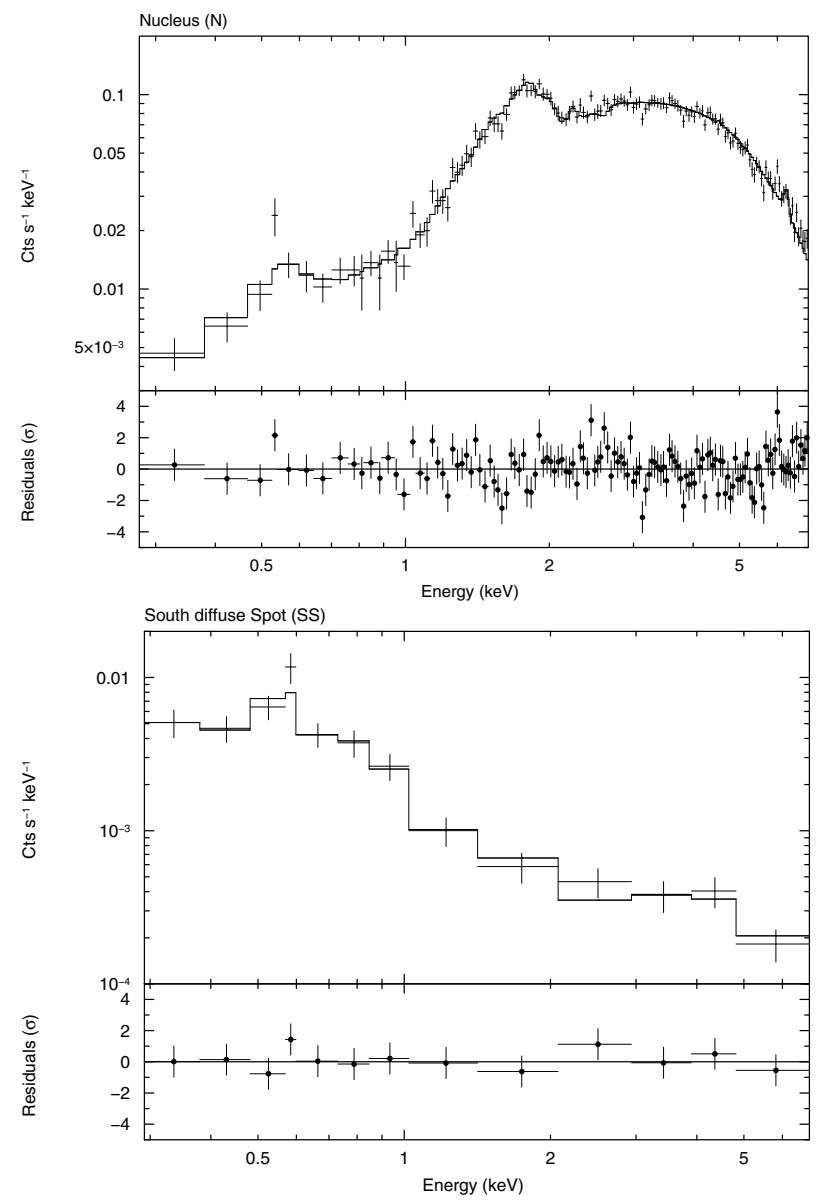
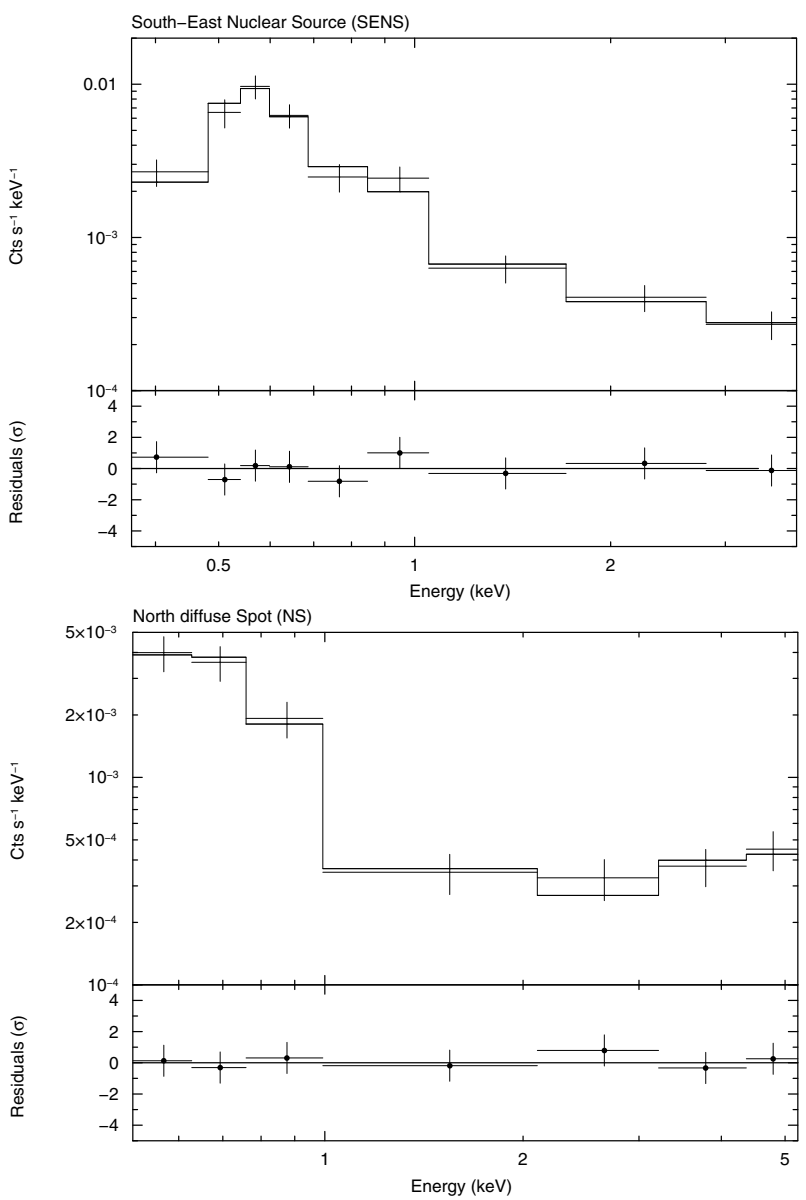

Fig. 4. Spectra (upper panels) and residuals in units of standard deviations (lower panels) for the four regions of NGC 5252 defined in Fig. 2.

Table 3. ACIS-S best-fit parameters and results for the spatially-resolved regions of NGC 5252.

\begin{tabular}{|c|c|c|c|c|c|c|c|c|c|c|}
\hline Region & $\begin{array}{c}N_{\mathrm{H}}{ }^{a} \\
\left(10^{22} \mathrm{~cm}^{-2}\right)\end{array}$ & $\Gamma^{b}$ & $\begin{array}{l}k T^{c} \\
(\mathrm{eV})\end{array}$ & $\begin{array}{c}E_{\mathrm{c}}{ }^{d} \\
(\mathrm{keV})\end{array}$ & $\begin{array}{l}E W^{e} \\
(\mathrm{eV})\end{array}$ & $F_{0.5-2 \mathrm{keV}^{f}}^{f}$ & $F_{2-10 \mathrm{keV}^{f}}$ & $L_{0.5-2 \mathrm{keV}^{g}}$ & $L_{2-10 \mathrm{keV}^{g}}^{g}$ & $\chi^{2} / v$ \\
\hline Nucleus & $2.32 \pm_{0.15}^{0.13}$ & $1.00 \pm_{0.06}^{0.08}$ & $140 \pm_{40}^{60}$ & $6.37 \pm_{0.05}^{0.06}$ & $50 \pm 20$ & $2.66 \pm_{0.28}^{0.11}$ & $103 \pm_{3}^{2}$ & $0.82 \pm_{0.09}^{0.03}$ & $1130 \pm_{30}^{20}$ & $176.9 / 127$ \\
\hline SENS & $0.47 \pm_{0.31}^{0.16}$ & $0.8 \pm 0.4$ & $<140$ & $\ldots$ & $\cdots$ & $0.06 \pm 0.05$ & $0.34 \pm_{0.14}^{0.08}$ & $50 \pm 40$ & $4.1 \pm_{1.7}^{1.0}$ & $3.0 / 5$ \\
\hline NS & $\equiv N_{\mathrm{H}, \mathrm{Gal}}$ & $-0.9 \pm_{0.6}^{0.5}$ & $240 \pm_{30}^{40}$ & $\ldots$ & ... & $0.066 \pm_{0.015}^{0.011}$ & $1.3 \pm 0.3$ & $0.89 \pm_{0.20}^{0.15}$ & $15 \pm 3$ & $1.0 / 3$ \\
\hline SS & $\equiv N_{\mathrm{H}, \mathrm{Gal}}$ & $0.0 \pm 0.4$ & $\begin{array}{c}110 \pm_{21}^{13} \\
510 \pm_{230}^{170}\end{array}$ & $\ldots$ & $\ldots$ & $0.122 \pm_{0.023}^{0.017}$ & $0.70 \pm_{0.53}^{0.18}$ & $1.7 \pm_{0.3}^{0.2}$ & $8 \pm_{6}^{2}$ & $5.1 / 7$ \\
\hline
\end{tabular}

Notes. ${ }^{(a)}$ Absorbing column density; ${ }^{(b)}$ photon index; ${ }^{(c)}$ temperature of the emitting thermal gas derived using mekal to fit the spatially resolved data; ${ }^{(d)}$ energy of the emission line; ${ }^{(e)}$ equivalent width; ${ }^{(f)}$ flux in units of $10^{-13} \mathrm{erg} \mathrm{s}^{-1} \mathrm{~cm}^{-2}$; ${ }^{(g)}$ luminosity in units of $10^{40} \mathrm{erg} \mathrm{s}^{-1}$.

\section{Comparing soft X-ray and [OIII] morphologies}

NGC 5252 was observed in the [OIII] band with the WFPC2 onboard HST on 1995, July 23, with the linear ramp filter FR533N. The data were downloaded from MAST (multi-mission archive at STScI). The images were processed through the standard OTFR (on-the-fly reprocessing) calibration pipeline which performs analog-to-digital conversion, bad pixel masking, bias and dark subtraction, flat field correction and photometric calibration. The cosmic ray rejection was performed combining the two images that are usually taken for this scope. Geometric distortion was corrected with the multi drizzle script (Koekemoer et al. 2002).

The relative Chandra-HST astrometry is clearly a fundamental issue for this work. Chandra has a nominal position accuracy of $0.6^{\prime \prime}$ while the absolute astrometry of HST is accurate to $1-2^{\prime \prime}$. Fortunately we could use a point-like source detected both in the WFPC2 and Chandra fields to align the two astrometric solutions. This source was previously detected at radio wavelengths (Wilson \& Tsvetanov 1994) and is most probably associated to a background quasar (Tsvetanov et al. 1996). Moreover we used the brightest emission peak in the nuclear region of NGC 5252 itself as a second reference point.

Images were calibrated in flux with a constant flux conversion factor of $1.839 \times 10^{-16}$, corresponding to the flux producing a count rate of $1 \mathrm{~s}^{-1}$ in the filter band. The above value is appropriate for the instrumental configuration employed during the NGC 5252 exposure, as indicated by the PHOTFLAM keyword in the image file. 

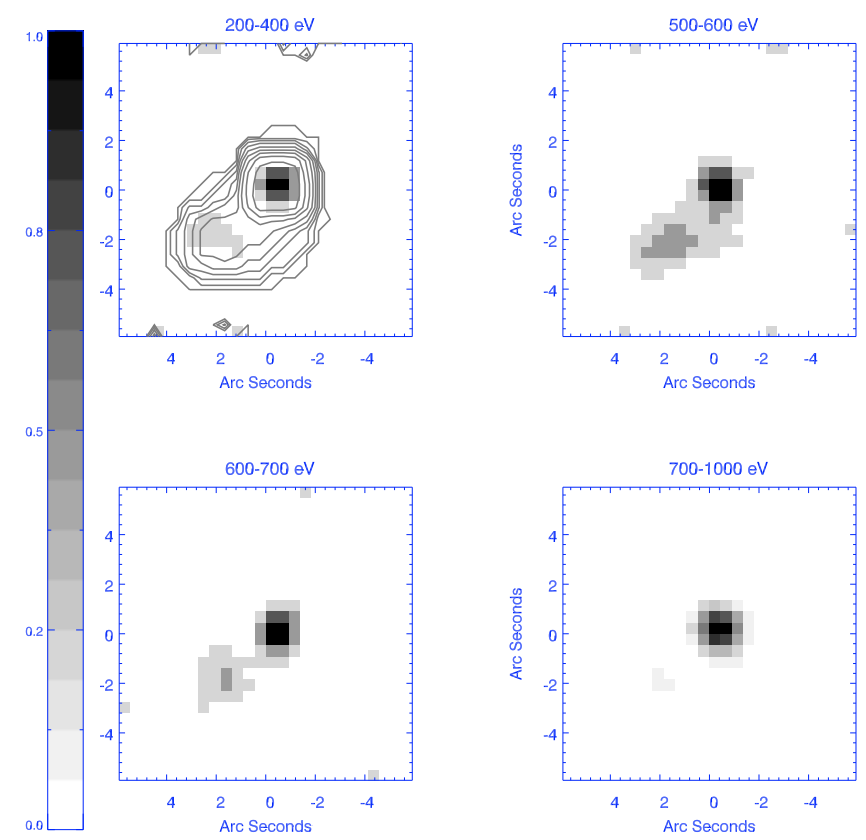

Fig. 5. Narrow-band ACIS-S images in the $10^{\prime \prime}$ around the NGC 5252 core normalized to the peak nuclear emission. Images are smoothed with a 5 pixel Gaussian kernel. The solid lines in the upper left panel represent a contour plot of the $0.2-1 \mathrm{keV}$ image, assuming the same smoothing criterion.

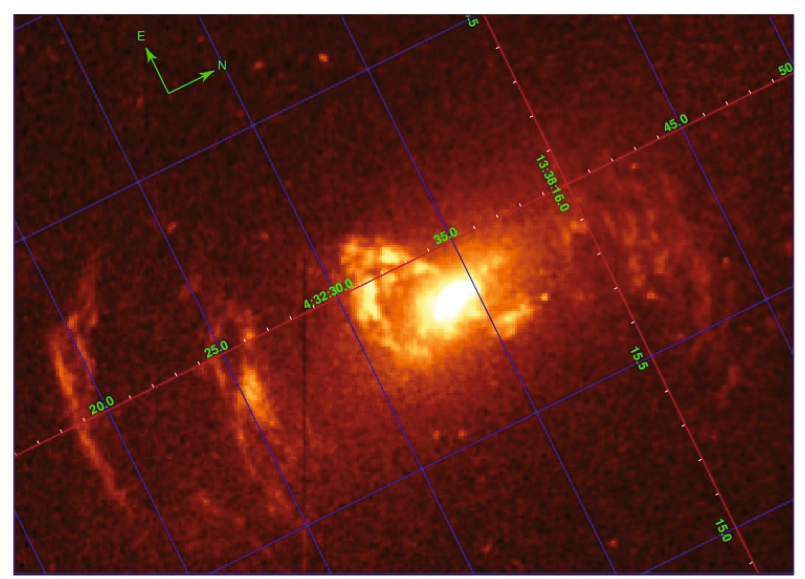

Fig. 6. HST WFC 2 [OIII] image of the ionisation cones in NGC 5252.

The HST image is shown in Fig. 6. Tsvetanov et al. (1996), Morse et al. (1998) and Capetti et al. (2005) discussed it in detail. We refer the reader to these papers for an extensive discussion on the properties of the optical emission. Their main outcomes can be summarized as follows:

- the surface brightness is dominated by the unresolved nucleus;

- a half-ring structure is apparent S-E of the nucleus at a maximum projected distance of $\simeq 1.5 \mathrm{kpc}$. It is probably associated with the near side of an inclined gas disc, whose far side is obscured by the host galaxy dust (Morse et al. 1998);

- the large scale ionisation cone is traced by thin shells of enhanced emission at either side of the nucleus, well aligned along a $\mathrm{PA} \simeq 110^{\circ}$ at distances between 5 and $11 \mathrm{kpc}$. Fainter co-aligned structures at scales as large as $20 \mathrm{kpc}$ are detected

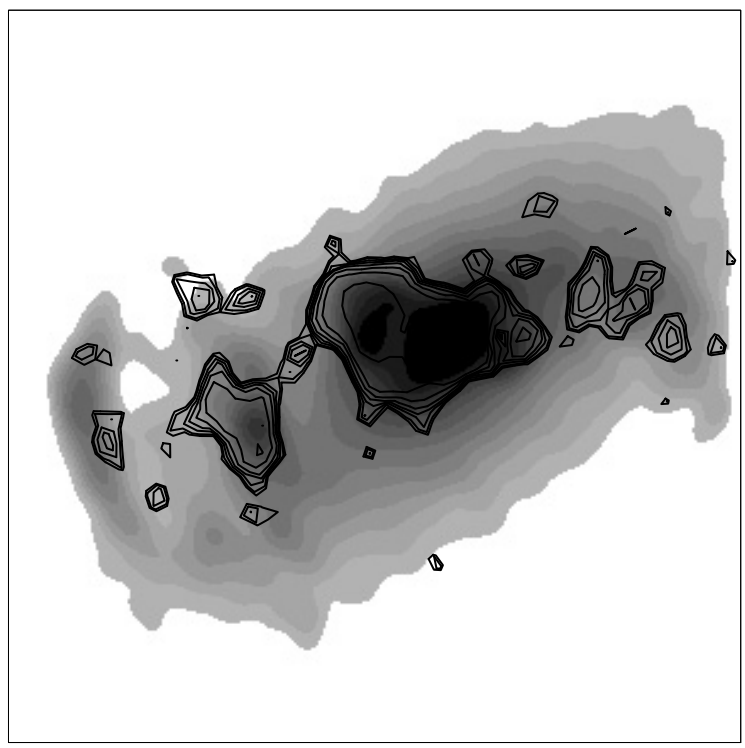

Fig. 7. Iso-intensity Chandra-ACIS $0.2-1 \mathrm{keV} X$-ray iso-intensity contours superposed to the HST WFC 2 [OIII] image of Fig. 6. The resolution of the latter was degraded to the typical resolution of Chandra optics by applying a wavelet smoothing with an 8 pixel kernel. The Chandra contours represent nine linearly space count levels from 0.5 to 20 counts per pixel, after a wavelet smoothing with a $\sigma=1.25$ pixel was applied.

as well in the $\mathrm{O}[\mathrm{III}]$ images; however, we will not discuss these latter structures, as they are beyond the region where X-ray emission associated with NGC 5252 is detected;

- there is no evidence of radial motions. The measured velocities of the different structures are fully explained by the rotations of the two discs [nonetheless Acosta-Pulido et al. (1996) claimed the detection of radial motions describing the kinematic properties of the [OIII] emission arcs].

In Fig. 7 we present the superposition between the soft X-rays iso-intensity contours to the [OIII] image. The HST image spatial resolution was degraded with an 8 pixel wavelet kernel to match the resolution of the Chandra optics. Regions of enhanced X-ray emission exhibit a remarkable coincidence with the morphology of the optical narrow-band image.

We calculated the ratio between the [OIII] band and the 0.5-2 keV flux (Fig. 8) for the regions specified after splitting region SS into two sub-regions divided by a E-W line at $\delta_{\mathrm{J} 2000}=4^{\circ} 32^{\prime} 21^{\prime \prime}$ (regions "SSNorth" - SSN - and "SSSouth" SSS - respectively). The ratio exhibits a dynamical range smaller than a factor 2 over distances ranging from less than $100 \mathrm{pc}$ to $\sim 1.5 \mathrm{kpc}$ with a slight tendency to decrease with the distance from the nucleus $(r)$. This last effect, however, is most probably an observational artifact due to the decreasing in surface brightness of the arcs moving away from the nucleus coupled with the sensitivity limits of Chandra to extended sources. At a first glance, the $[\mathrm{OIII}] /$ soft-X ratio profile as a function of the distance from the nucleus seems to suggest that the electron density follows a $r^{-2}$ relation because the number of ionising photons and of the overall average ionisation state of the nuclear species remains almost constant. This result agrees with Bianchi et al. (2006) who assumed a very simplified geometry of the emitting gas though. A more detailed investigation on this topic in NGC 5252 is hampered by the quality of the data. 


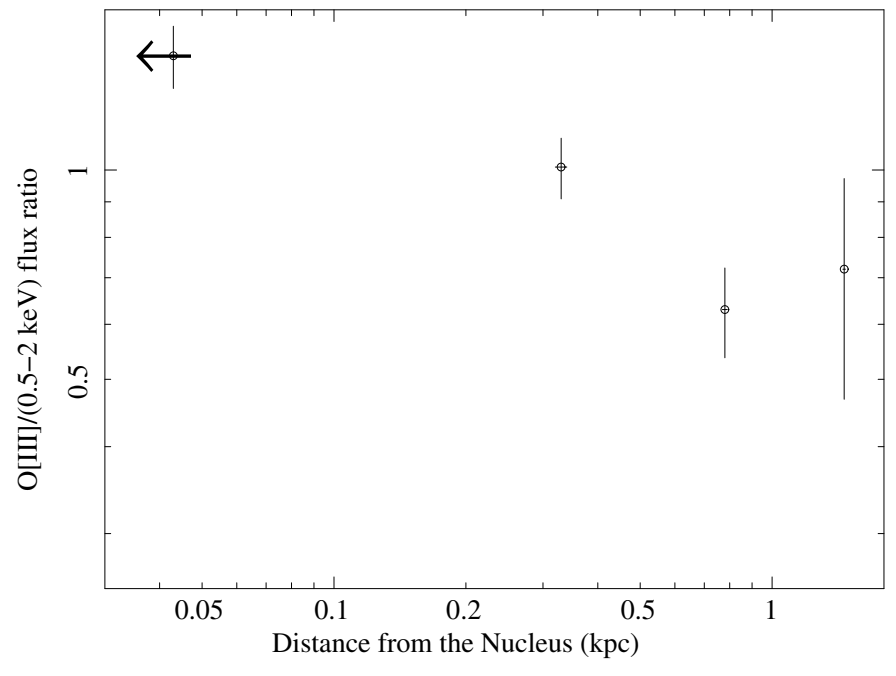

Fig. 8. $[\mathrm{OIII}] /$ soft-X flux ratio as a function of distance from the nucleus.

\section{Discussion}

The soft X-ray emission of NGC 5252 is clearly extended and the ACIS images demonstrate that the spectacular ionisation cones observed in [OIII] have counterparts in the $0.1-1 \mathrm{keV}$ band. The cumulative soft X-ray spectrum observed by XMMNewton is described by a soft power-law ( $\Gamma \sim 3)$. The ACIS images suggest that this is probably due to a blend of emission lines that mimics this steep power-law as demonstrated in other type II Seyferts like NGC 1068, Circinus galaxy and Mrk 3 (Kinkhabwala et al. 2002; Brinkman et al. 2002; Ogle et al. 2003; Sambruna et al. 2001; Sako et al. 2000; Bianchi et al. 2005; Pounds \& Page 2005). This scenario is also supported by the detection in the RGS high resolution spectrum of three emission lines, of CV, OVII and OVIII, probably due to photoionised gas. This is also consistent with previous optical studies that excluded collisional ionisation along the cones of NGC 5252 (Tsvetanov et al. 1996). Moreover, the presence of in situ ionisation sources due to shocks formed by large scale outflows interacting with the interstellar matter has been excluded (Morse et al. 1998). Thus the source of ionising photons is most probably the nucleus. With this assumption, we can use the imaging of the arcs to study the physical condition of the gas along the ionisation cones. In particular, the constant of $[\mathrm{OIII}] /(0.5-2 \mathrm{keV})$ flux ratio along the ionisation cones within the inner ${ }^{2} \sim 1.5 \mathrm{kpc}$ suggests a $r^{-2}$ law for the ion density.

Optical spectroscopic studies (Acosta-Pulido et al. 1996) suggest that the radial dependency of the ionisation parameter ${ }^{3}$ $U$ follows a different law in the south-east $\left(U \propto r^{-0.4}\right)$ with respect to the north-east $\left(U \propto r^{0}\right)$ cone. The authors speculate that the intrinsic behaviour should be the one shown in the former, while the radial-independence of the ionisation parameter in the latter may be due to a "conspiracy" introduced by the existence of two counterotating discs of gas (Morse et al. (1998): one is coplanar to the stellar one and the other is inclined by $\sim 40^{\circ}$.

\footnotetext{
2 The outer arcs and filaments (Tadhunter \& Tsvetanov 1989) are most probably too weak to be detected in X-rays. Considering the extension of the outer [OIII] arcs, the minimum detectable flux between $0.1-1 \mathrm{keV}$ is $F_{0.1-1 \mathrm{keV}} \sim 5 \times 10^{-15} \mathrm{erg} \mathrm{s}^{-1} \mathrm{~cm}^{-2}$ while assuming a constant $[\mathrm{OIII}] /$ soft X-ray ratio, the expected flux should be $\sim 10$ times lower.

${ }^{3} U=\frac{L}{4 \pi \rho r^{2}}$, where $L$ is the source's luminosity, $\rho$ is the density of the ionised gas, $r$ is the distance between the source of ionising photons and the ionised matter.
}

Morse et al. (1998) speculated that the prominence of the southeast [OIII] cone in the nuclear regions is caused by this component being seen directly, while the northeast [OIII] cone is seen through the gas of the other disc. If that is the case, the absorption due to this component could alter the line ratios presented by Acosta-Pulido et al. (1996) and thus the correct behaviour of $\mathrm{U}$ should be the one derived from the south-east cone. $U \propto r^{-0.4}$ implies that the luminosity of the nucleus increased by a factor $\Delta L \sim 3-6$ in the last $\Delta t \sim 5000$ years. These numbers become $\Delta L \sim 10-30$ and $\Delta t \sim 30000$ years if we further assume that the $\mathrm{U}$ and the ion density laws are still valid up to $10 \mathrm{kpc}$ from the nucleus, i.e. where the optical cones are still detectable in [OIII] but not in X-rays.

On the contrary, having $U$ constant and $\rho \propto r^{-2}$ would imply that $\mathrm{L}$ has remained constant during the last 5000 (30 000) years. This is consistent with the "quasar-relic" scenario proposed by Capetti et al. (2005). These authors suggested that the nucleus of NGC 5252 is indeed the "relic" of a nucleus that already experienced the activity phase in the past and that now persists in an almost quiescent phase. This is suggested by the high mass of the $\operatorname{SMBH}\left(M_{\mathrm{BH}}=10^{9} M_{\odot}\right.$, Capetti et al. 2005) that indicates that the nucleus has already accreted in the past, the low Eddington ratio $\left(L \sim 10^{-3} L_{\mathrm{Edd}}\right.$, assuming the bolometric correction from Marconi et al. (2004), $\left.L_{\mathrm{hard}-\mathrm{x}} \sim(1 / 22) \times L_{\mathrm{bol}}\right)$, and the early type (S0) morphology of the AGN host galaxy. In the literature it is also reported that the optical emission line ratios in the inner 30" are typical of LINERS (Goncalves et al. 1998), thus suggesting that a low efficiency engine is acting at the nucleus of the source. It is worth noting that also the detection of two counterotating discs suggests that NGC 5252 is a "quasar-relic". These discs are tracers of a major merging event that occurred most probably more than $10^{8}$ years ago, because the stellar disc of NGC 5252 is undisturbed. If the merging event triggered a phase of AGN activity (see Jogge 2006, and references therein for a discussion of this topic), we can expect that it lasted few/some $\sim 10^{7}$ years (Martini \& Weinberg 2001; Steidel et al. 2002; Jakobsen et al. 2003; Gonçalves et al. 2008) after which the source has persisted in a quiescent state.

Finally it is worth noting that the spectrum of the nucleus hosted by NGC 5252 is confirmed to be quite flat (Cappi et al. 1996). As shown, if modelled with a simple absorbed power-law its photon index points to a very hard spectrum $(\Gamma \sim 1)$. The low $E W(\sim 50 \mathrm{eV})$ of the neutral $\left(E_{\mathrm{FeK} \alpha} \sim 6.4 \mathrm{keV}\right)$ iron line is consistent with what is expected if the $\mathrm{FeK} \alpha$ line is produced via transmission in the observed column $\left(N_{\mathrm{H}} \sim 2 \times 10^{22} \mathrm{~cm}^{-2}\right.$, Makishima 1986) thus excluding a reflection dominated spectrum. To reconcile the hardness of the NGC 5252 nuclear spectrum at least marginally, with previous results for Seyfert galaxies $(\Gamma \sim 1.5-2.5$; Turner \& Pounds 1989; Nandra \& Pounds 1994; Smith \& Done 1996; Dadina 2008), we must invoke complex absorption models involving partial covering of the source and/or ionised absorbers along the line-of-sight. In this case the spectral index becomes $\Gamma \sim 1.4-1.5$. It is remarkable that the flat photon index may be a further clue suggesting that the X-rays may be produced in an ADAF (Narayan \& Yi 1994) as expected in a "quasar-relic".

Acknowledgements. This paper is based on observations obtained with XMM-Newton, an ESA science mission with instruments and contributions directly funded by ESA Member States and the USA (NASA). M.D. greatfully acknowledges Barbara De Marco for helpful discussions. M.D., M.C. and G.M. greatfully acknowledge ASI financial support under contract I/23/05/0. C.V. greatfully acknowledges ASI financial support under contract I/088/06/0. 


\section{References}

Acosta-Pulido, J. A., Vila-Vilaro, B., Perez-Fournon, I., Wilson, A. S., \& Tsvetanov, Z. I. 1996, ApJ, 464, 177

Antonucci, R. 1993, ARA\&A, 31, 473

Baskin, A., \& Laor, A. 2005, MNRAS, 358, 1043

Bianchi, S., Miniutti, G., Fabian, A. C., \& Iwasawa, K. 2005, MNRAS, 360, 380

Bianchi, S., Guainazzi, M., \& Chiaberge, M. 2006, A\&A, 448, 499

Bianchi, S., Chiaberge, M., Evans, D. A., et al. 2010, MNRAS, 405, 553

Brinkman, A. C., Kaastra, J. S., van der Meer, R. L. J., et al. 2002, A\&A, 396, 761

Capetti, A., Marconi, A., Macchetto, D., \& Axon, D. 2005, A\&A, 431, 465

Cappi M., Mihara T., Matsuoka M., et al. 1996, ApJ, 458, 149

Cappi M., Panessa F., Bassani L., et al. 2006, A\&A, 446, 459

Dadina, M. 2007, A\&A, 461, 1209

Dadina M. 2008, A\&A, 485, 417

der Herder J., Brinkman A. C., Kahn S. M., et al. 2001, A\&A, 365, L7

de Vaucouleurs, G., de Vaucouleurs, A., Corwin, H. G., Jr., et al. 1991, Third Reference Catalogue of Bright Galaxies (New York: Springer)

Dickey, J. M., \& Lockman, F. J. 1990, ARA\&A, 28, 215

Gabriel A.H., \& Jordan C. 1969, MNRAS, 145, 241

Gabriel C., Denby M., Fyfe D. J., Hoar J., \& Ibarra A. 2003, in Astronomical Data Analysis Software and Systems XIII, ed. F. Ochsenbein, M. Allen, \& D. Egret (San Francisco: ASP), ASP Conf. Ser., 314, 759

Goncalves, A. C., Veron, P., \& Veron-Cetty, M.-P. 1998, A\&A, 333, 877

Gonçalves, T. S., Steidel, C. C., \& Pettini, M. 2008, ApJ, 676, 816

Guainazzi M., \& Bianchi S. 2006, MNRAS, 374, 1290

Guainazzi M., Risaliti G., Nucita A., et al. 2009, A\&A, 505, 589

Jakobsen, P., Jansen, R. A., Wagner, S., \& Reimers, D. 2003, A\&A, 397, 891

Jogee, S. 2006, Physics of Active Galactic Nuclei at all Scales, 693, 143
Kinkhabwala A., Sako M., Behar E., et al. 2002, ApJ, 575, 732

Koekemoer, A. M., Fruchter, A. S., Hook, R. N., \& Hack, W. 2002, in The 2002 HST Calibration Workshop, ed. S. Arribas, A. Koekemoer, \& B. Whitmore (Baltimore, MD: Space Telescope Science Institute), 339

Makishima, K. 1986, The Physics of Accretion onto Compact Objects, 266, 249 Marconi, A., \& Hunt, L. K. 2003, ApJ, 589, L21

Marconi A., Risaliti G., Gilli R., et al. 2004, MNRAS, 351, 169

Martini, P., \& Weinberg, D. H. 2001, ApJ, 547, 12

McLure, R. J., \& Dunlop, J. S. 2001, MNRAS, 327, 199

Menci, N., Fiore, F., Perola, G. C., \& Cavaliere, A. 2004, ApJ, 606, 58

Mewe R., Gronenschild E. H. B. M., \& van der Oord G. H. J. 1985, A\&AS, 62, 197

Morse J.A., Cecil G., Wilson A. S., \& Tsvetanov Z. I. 1998, ApJ, 505, 159

Nandra, K., \& Pounds, K. A. 1994, MNRAS, 268, 405

Narayan, R., \& Yi, I. 1994, ApJ, 428, L13

Ogle, P. M., Brookings, T., Canizares, C. R., Lee, J. C., \& Marshall, H. L. 2003, A\&A, 402, 849

Osterbrock, D. E., \& Martel, A. 1993, ApJ, 414, 552

Porquet, D., \& Dubau J. 2000, A\&AS, 143, 495

Pounds, K. A., \& Page, K. L. 2005, MNRAS, 360, 1123

Risaliti G. 2002, A\&A, 386, 379

Sako, M., Kahn, S. M., Paerels, F., \& Liedahl, D. A. 2000, ApJ, 543, L115

Sambruna, R. M., Brandt, W. N., Chartas, G., et al. 2001, ApJ, 546, L9

Smith, D. A., \& Done, C. 1996, MNRAS, 280, 355

Steidel, C. C., Hunt, M. P., Shapley, A. E., et al. 2002, ApJ, 576, 653

Tadhunter, C., \& Tsvetanov, Z. 1989, Nature, 341, 422

Tsvetanov, Z. I., Morse, J. A., Wilson, A. S., \& Cecil, G. 1996, ApJ, 458, 172

Turner, T. J., \& Pounds, K. A. 1989, MNRAS, 240, 833

Turner T. J., George I. M., Nandra K., \& Mushotzky R. F. 1997, ApJS 113, 23

Wilson, A. S., \& Tsvetanov, Z. I. 1994, AJ, 107, 1227 